\title{
Colopexia a través de celiotomía como tratamiento del prolapso rectal recidivante en gatos
}

\section{Colopexy through celiotomy as treatment of recurring rectal prolapse in cats}

\author{
Ivan Camilo Sánchez R..$^{1,3}$, Catherin Lorena Solarte J. ${ }^{1}$, Marco Antonio Virgen L. ${ }^{2}$
}

\section{Resumen}

Se presenta el caso de dos gatos con prolapso rectal recidivante; macho y hembra de 3 y 5 meses de edad, respectivamente. En la exploración clínica se evidenció dos pacientes con condición corporal $2 / 5$, mucosas pálidas, deshidratación y prolapso de aproximadamente $6 \mathrm{~cm}$ de recto con presencia de estructuras larvarias de mosca doméstica. El tutor refiere que un mes atrás a la consulta, los gatos fueron rescatados y se les practicó reducción digital con sutura en ano como medida correctiva de un primer prolapso rectal que recidivó transcurridos quince días de dicha intervención. Como medida correctiva urgente, y previa estabilización de los pacientes, se practicó colopexia a través de celiotomía empleando para tal fin poliglactina 910. No se presentaron complicaciones intra y posoperatorias. Durante 12 meses ulteriores a la colopexia no hubo recidiva del prolapso en ninguno de los pacientes.

Palabras clave: prolapso rectal, colopexia, gatos, poliglactina 910

\footnotetext{
${ }^{1}$ Medivet Centro Veterinario, Mocoa, Putumayo Colombia

${ }^{2}$ Facultad de Ciencias Agropecuarias, Universidad de la Amazonía, Florencia, Caquetá, Colombia

${ }_{3}^{3}$ E-mail: i.sanchez@udla.edu.co
}

Recibido: 30 de enero de 2021

Aceptado para publicación: $x x x$

Publicado: 24 de agosto de 2021

CLos autores. Este artículo es publicado por la Rev Inv Vet Perú de la Facultad de Medicina Veterinaria, Universidad Nacional Mayor de San Marcos. Este es un artículo de acceso abierto, distribuido bajo los términos de la licencia Creative Commons Atribución 4.0 Internacional (CC BY 4.0) [https:// creativecommons.org/licenses/by/4.0/deed.es] que permite el uso, distribución y reproducción en cualquier medio, siempre que la obra original sea debidamente citada de su fuente original 
The case of two cats with recurrent rectal prolapse is presented; 3- and 5-month-old male and female, respectively. The clinical examination revealed two patients with body condition $2 / 5$, pale mucous membranes, dehydration and a prolapse of approximately 6 $\mathrm{cm}$ of the rectum with the presence of housefly larval structures. The tutor reports that a month before the consultation, the cats were rescued and underwent digital reduction with suture in the anus as a corrective measure for a first rectal prolapse that recurred fifteen days after said intervention. As an urgent corrective measure, and after stabilizing the patients, a colopexy was performed through celiotomy, using polyglactin 910 for this purpose. There were no intraoperative and postoperative complications. During 12 months after colopexy, there was no recurrence of prolapse in any of the patients.

Key words: Rectal prolapse, colopexy, cats, polyglactin 910

\section{INTRODUCCIÓN}

El prolapso rectal se define como la protrusión o inversión del recto, o de algunas de sus capas, más allá del borde anal (Oliveira et al., 2009). Puede ocurrir en humanos y en otras especies animales (Goodall et al., 2018). En gatos, el prolapso rectal se puede presentar en individuos de cualquier edad, raza o sexo, aunque con una mayor probabilidad en jóvenes con diarrea y tenesmo (Cunha et al., 2015).

La patogénesis del prolapso rectal está relacionada con la debilidad de los músculos del piso pélvico, puborrectal y esfínter interno y externo (Bustamante et al., 2016). No obstante, algunas etiologías como la urolitiasis, prostatitis, leiomioma rectal, cuerpos extraños y colitis bacteriana y parasitaria han sido asociadas a estas alteraciones anatómicas y, por ende, señaladas como las principales causas del prolapso rectal en gatos (Goodall et al., 2018).

Sanchez et al. (2017) y Williams (2009) discuten acerca del tratamiento y mencionan que ante un buen aspecto del prolapso se realiza reducción digital acompañando con sutura en bolsa de tabaco, en tanto que cuando la mucosa rectal aparece desvitalizada se recurre a la resección de la porción y en casos de recidivas luego de una reducción digital se sugiere la corrección mediante colopexia a través de celiotomía o laparotomía. La colopexia, cuya etimología indica colo -colon- pexis -fijación-, se refiere al acto quirúrgico en el que se crea una adhesión permanente entre el colon descendente y la pared abdominal con el objetivo de evitar desplazamientos caudales (Brun et al., 2004). Czajkowski y Fryer (2020), de otra parte, manifiestan que la colopexia está indicada en animales de compañía en casos de prolapso rectal recidivante, hernia perineal y torsión de colon. Esta técnica puede realizarse mediante abordaje por celiotomía o laparoscopia (Torrijo et al., 2018).

La técnica de colopexia, como medida correctiva del prolapso rectal recidivante, es descrita por Smeak (2020), quien menciona que se debe acceder a la cavidad peritoneal, identificar el colon y halar hacia craneal para introducir la porción protruida; posteriormente, el colon es fijado mediante sutura al músculo transverso abdominal. Algunas consideraciones quirúrgicas relevantes sugieren que la sutura solamente debe perforar la serosa del colon y en ninguna circunstancia atravesar la luz de este (Brun et al., 2004) 

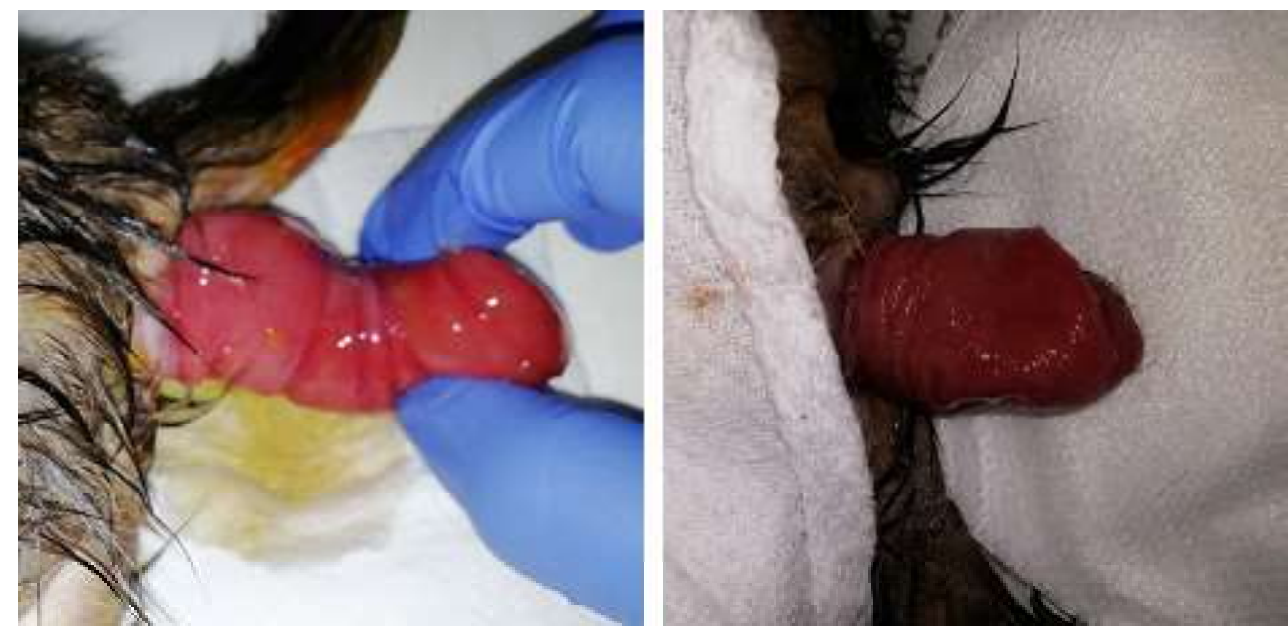

Figura 1. Prolapso rectal en gata de 5 meses (izquierda) y en gato de 3 meses (derecha)

El presente artículo tiene como objetivo informar dos casos de prolapso rectal recurrente en gatos cuya corrección definitiva se llevó a cabo mediante colopexia a través de celiotomía.

\section{Presentación del Caso}

\section{Reseña y motivo de consulta}

Fueron remitidos a consulta veterinaria dos gatos (Felis silvestris gatus) - macho y hembra - con edades de 3 y 5 meses y 0.45 y $1.0 \mathrm{~kg}$ de peso, respectivamente, quienes presentaban diarrea y protrusión de aproximadamente $6 \mathrm{~cm}$ de la estructura rectal (Figura 1).

\section{Anamnesis}

En conversación con el tutor, este reportó que los dos gatos habían sido rescatados de la calle un mes atrás e inmediatamente llevados a otro centro veterinario porque los animales «tenían el intestino salido por la cola». El diagnóstico emitido por el veterinario fue prolapso rectal y el protocolo instaurado consistió en vermifugación con $30 \mathrm{mg} / \mathrm{kg}$ de pirantel pamoato en dosis única y reducción digital acompañada de sutura en bolsa de tabaco en el ano.

\section{Exploración clínica y diagnóstico}

El propedéutico de los pacientes mostró constantes fisiológicas normales, tiempo de llenado capilar 3 segundos y turgencia de piel 4 segundos. En análisis coprológico mostró alta infestación por Toxocara sp y la biometría hemática indicó ligera anemia y leucocitosis en ambos pacientes. Durante la inspección y olfacción se pudo apreciar una coloración rosada de la estructura protruida, residuos de pelos y tierra adheridos, larvas de mosca doméstica de $2 \mathrm{~mm}$ de diámetro sobre la superficie y olor fétido. A partir de los hallazgos de la exploración clínica y anamnesis se dio como diagnóstico definitivo prolapso rectal recurrente en los dos gatos y se escogió la colopexia como medida correctiva.

\section{Tratamiento}

La terapéutica empleada para la diarrea consistió en hidratación intravenosa con lactato de Ringer, metronidazol (Metronidazol $\left.{ }^{\circledR}\right) 30 \mathrm{mg} / \mathrm{kg}$ PO c/12 h/5 días, 

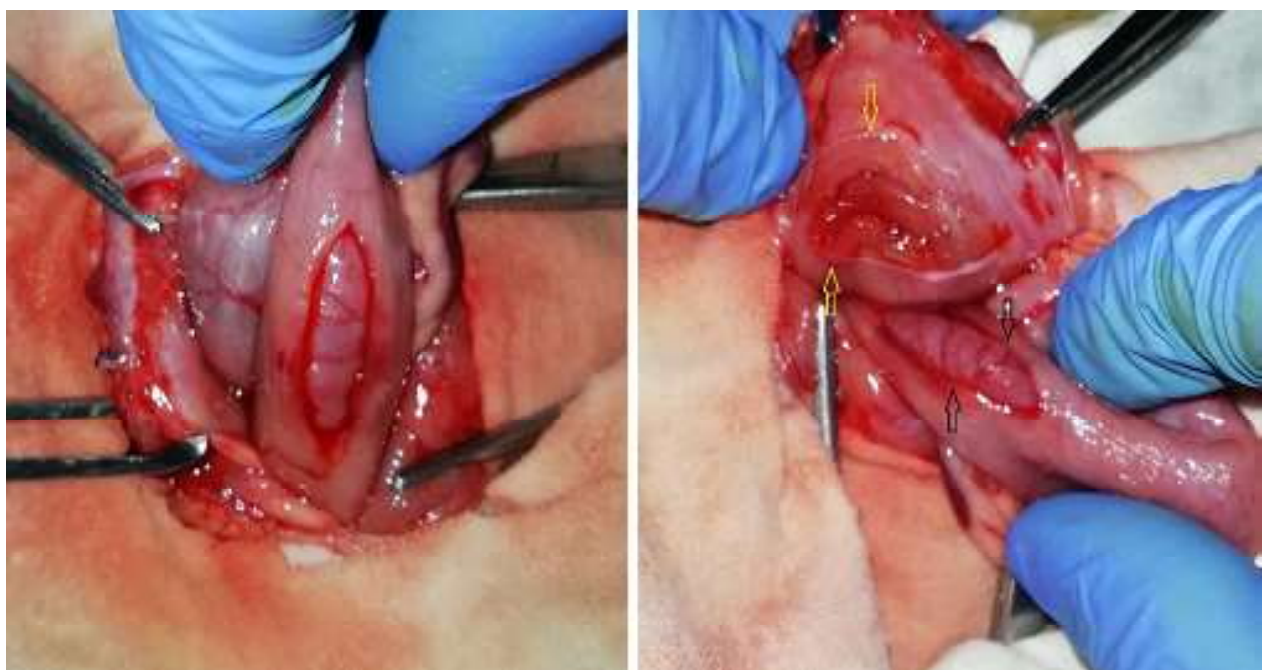

Figura 2. Colopexia a través de celiotomía en gatos con prolapso rectal. Izquierda (A): Incisión sobre la capa serosa del colon. Derecha (B): Delimitación de los cortes en el músculo transverso abdominal (flecha amarilla) y colon (flecha negra)
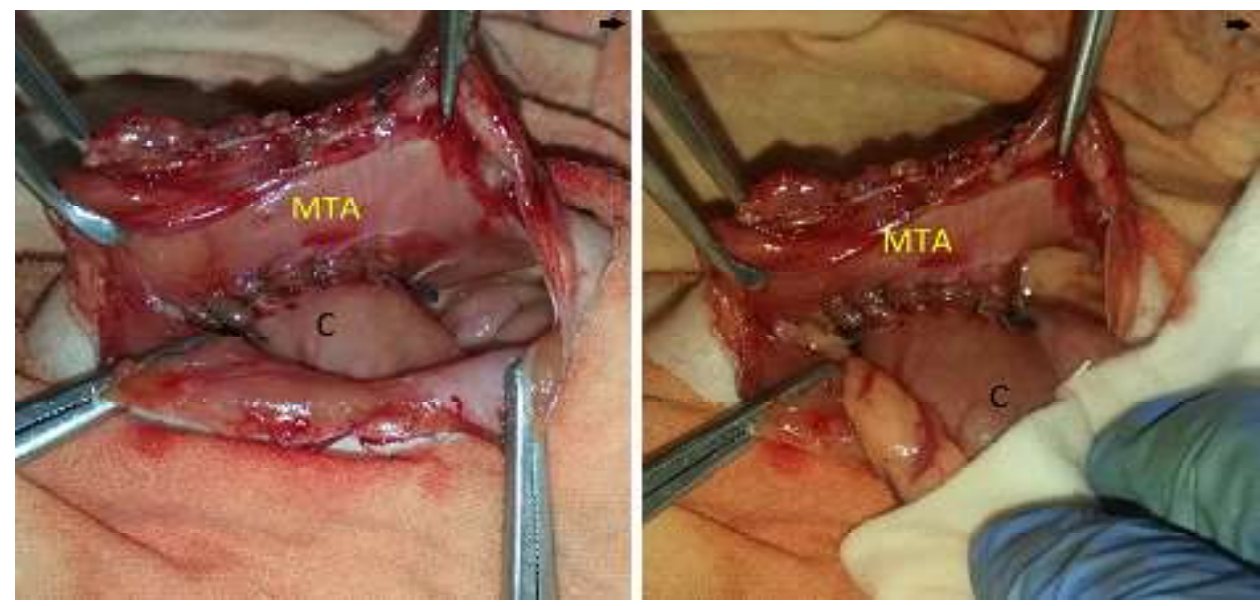

Figura 3. Colopexia realizada mediante patrón de sutura simple continua. Se aprecia la intersección que da lugar a la fijación del colon al músculo transverso abdominal. Las flechas negras (parte superior derecha) indican la ubicación de la cabeza de los gatos

trimetoprim + sulfa $\left(\right.$ Sutrim $\left.{ }^{\circledR}\right) 30 \mathrm{mg} / \mathrm{kg}$ PO c/24 h/5 días; y se acompañó con vermifugación de amplio espectro empleando Milbemicina + Praziquantel (Milpro®) $3.5 \mathrm{mg} /$ $\mathrm{kg}$ PO dosis única.

\section{Técnica quirúrgica colopexia}

Previa estabilización, los pacientes fueron sometidos a tiempo de ayuno sólido y líquido de 12 horas. El control antibiótico y 
analgésico se realizó con cefalexina (Rilexine $\left.{ }^{\circledR}\right) 25 \mathrm{mg} / \mathrm{kg} \mathrm{IM} \mathrm{c/12} \mathrm{h/5} \mathrm{días} \mathrm{y} \mathrm{ketopro-}$ feno (Prosyfen ${ }^{\circledR}$ ) $1 \mathrm{mg} / \mathrm{kg} \mathrm{SC} \mathrm{c} / 24 \mathrm{~h} / 3$ días. En premedicación se empleó xilacina (Xilasyn ${ }^{\circledR}$ ) $0.1 \mathrm{mg} / \mathrm{kg}$ IM y atropina (Atropina Zoo $\left.{ }^{\circledR}\right) 0.04 \mathrm{mg} / \mathrm{kg}$ IM. Para la inducción a la anestesia fija se utilizó tiletamina + zolazepam (Zoletil®) $5 \mathrm{mg} / \mathrm{kg}$ IM. Durante la intervención quirúrgica se mantuvo acceso venoso permanente mediante cateterización de la vena cefálica con catéter calibre 26. Previo al acto quirúrgico se higienizó el prolapso rectal empleando una solución de yodo y clorhexidina. Se ubicaron los pacientes en posición decúbito supino y se practicó tricotomía y embrocado de la región abdominal desde la apófisis xifoides hasta la ingle.

El intraoperatorio se inició con celiotomía de $4 \mathrm{~cm}$ de longitud sobre la línea alba a la altura del ombligo. Al ingresar a la cavidad peritoneal, se identificó el colon y se haló suavemente hacia craneal hasta conseguir la introducción del prolapso rectal. Con ayuda de un escalpelo se practicó una incisión superficial de aproximadamente $3 \mathrm{~cm}$ de longitud sobre el músculo transverso abdominal (MTA) del flanco derecho, procurando no atravesar la estructura muscular. El corte se realizó en dirección craneal-caudal paralela a la línea alba a una distancia de $2 \mathrm{~cm}$ de la celiotomía. Luego se efectuó una incisión de $3 \mathrm{~cm}$ de longitud en la capa serosa del colon sobre la cara lateral (Figura 2A); el corte fue iniciado con escalpelo y apoyado con tijera Mayo roma para no irrumpir sobre las demás capas y/o atravesar la luz del colon. Una vez obtenidos los cortes simétricos en colon y MTA (Figura 2B) se procedió a confrontar los cuatro bordes para fijar la estructura.

Para la fijación -pexis- se empleó material de sutura absorbible del tipo poliglactina 910 calibre 3-0. La fijación se logró aplicando suturas simples continuas sobre los bordes inferiores y posteriores de las estructuras (Figura 4). Luego de realizar la colopexia, se hizo un lavado peritoneal con suero fisiológico. Para el cierre del músculo se empleó sutura entrelazada con material absorbible, el tejido subcutáneo con puntos continuos y la piel con puntos simples separados de material no absorbible tipo polipropileno.

No se presentaron complicaciones durante el desarrollo de las cirugías de colopexia por celiotomía en los dos pacientes. La duración promedio de los procedimientos fue de 38 minutos. Se realizó seguimiento durante 12 meses ulteriores a la colopexia en los cuales no se presentaron recidivas del prolapso rectal en los gatos.

\section{Resultados y Discusión}

El prolapso rectal es una condición poco común en animales pequeños. Se trata de un trastorno del piso pélvico en el cual el recto pierde su estabilidad a causa del debilitamiento del tejido conectivo -encargado de su anclaje al sacro- y el músculo puborrectal (Barrand, 1999). Los prolapsos rectales ocasionan menoscabo sobre la calidad de vida de los gatos, y si no se corrigen a tiempo pueden conducir a úlcera rectal y sepsis (Torrijo et al., 2018). No existen reportes acerca de la incidencia del prolapso rectal en gatos, pero en humanos se indica una frecuencia de 2.5 por 100000 habitantes (Rockbrand et al., 2019).

Las patologías subyacentes asociadas comúnmente al prolapso rectal en gatos incluyen diarrea, parasitismo y tenesmo (Smeak, 2020). No obstante, otras enfermedades tales como divertículo uraco-vesical (Cunha et al., 2015), linfoma de unión ileocecocólica (Demetriou y Welsh, 1999), carcinoma de células de transición de la vejiga urinaria (Barrand, 1999) y ensanchamiento del orificio anal (Corgozinho et al., 2010) han sido reportadas como causas inusuales de prolapso rectal en gatos.

La implementación de la colopexia como medida correctiva del prolapso rectal recidivante en gatos ha sido reportada por Jayaprakash et al. (2010). Esta técnica también ha sido informada en ovejas (Oliveira et 
al., 2009), yeguas (Broyles et al., 2018), perros (Hernández, 2010) y primates (Goodall et al., 2018). La colopexia no interfiere con la función intestinal de los gatos; las complicaciones derivadas de la técnica quirúrgica son relativamente nulas; aun así, atravesar la luz del colon o ejercer mucha tensión sobre este al momento de fijar con la sutura pueden representar los únicos inconvenientes posoperatorios (Popovitch et al., 1994).

No se dispone de informes sobre la tasa de recidiva de prolapso rectal posterior a colopexia en gatos; sin embargo, Bustamante et al. (2016) afirman que para asegurar el éxito de la técnica es necesario corregir la causa subyacente del prolapso. Por su parte, Bergamaschi y Corman (2014) manifiestan que el porcentaje de recurrencia en humanos fue de $7 \%$ a los 5 años y $29 \%$ a los 10 años de realizada la colopexia.

Corgozinho et al. (2010) informó de dos casos de recidiva de prolapso rectal luego de colopexia en gatos; no obstante, la protrusión del recto en dichas casuísticas era ocasionada por ensanchamiento del orificio anal, por lo tanto, fue necesaria la colocación de una cinta de elastómero de silicona alrededor del ano para conseguir la remisión definitiva de los prolapsos rectales. Esto ratifica las afirmaciones de Bustamante et al. (2016) sobre la importancia de eliminar las causas subyacentes del prolapso para obtener resultados satisfactorios con la colopexia.

Sobre la causa subyacente del prolapso rectal de los dos gatos cachorros reportados en el presente artículo, se pudo constatar mediante el examen físico y paraclínico que la infestación por Toxocara sp fue la causa desencadenante y condujo a la recidiva de los prolapsos corregidos inicialmente con sutura en bolsa de tabaco. Estos resultados coinciden con Secchi et al. (2012) quienes señalan a la toxocariosis como causante de prolapso rectal en una gata adulta.
En el seguimiento realizado durante un año al acto quirúrgico no se presentaron complicaciones relacionadas con la cirugía. Los autores del presente artículo se refieren a la colopexia a través de celiotomía como una técnica asequible con resultados altamente satisfactorios siempre que la causa desencadenante del prolapso, para este caso la toxocariosis, haya sido resuelta totalmente. Además, la colopexia resulta una técnica quirúrgica muy versátil: el abordaje puede realizarse a través de laparotomía o celiotomía (Czajkowski y Fryer, 2020) y es posible emplear poliglactina 910 o polipropileno para la fijación del colon (Brun et al., 2004); todo en función de los materiales y equipos disponibles para tal fin.

\section{Literatura Citada}

1. Barrand KR. 1999. Rectal prolapse associated with urinary bladder neoplasia in a cat. J Small Anim Pract 40: 222223. doi: $10.1111 /$ j.1748-5827.1999.tb03065.x

2. Bergamaschi R, Corman M. 2014. El dilema del prolapso rectal: abundancia de adjetivos. Cir Esp (Engl Ed) 92: 147-148. doi: 10.1016/j.ciresp.2013.12.003

3. Broyles A, Hopper S, Woodie B, Ruggles A. 2018. Clinical outcomes after colopexy through left ventral paramedian incision in 156 thoroughbred broodmares with large colon disorders (1999-2015). Vet Surg 47: 490-498. https://doi.org/10.1111/vsu.12791

4. Brun M, Pippi N, Beck C, Contesini E, Pereira R, Stedile R, Bonfada A. Bordin A, et al. 2004. Avaliação de dois diferentes fios de sutura para colopexia incisional laparoscopia em cães: estudo experimental. Braz J Vet Res Anim Sci 41: 154-161. doi: 10.1590/s1413-95962004000300002 
5. Brun M, Pippi N, Beck C, Contesini E, Pereira R, Stedile R, Bonfada A, Columé L, et al. 2004. Colopexia incisional por celiotomia ou transparietal auxiliada por laparoscopia em cães. Cienc Rural 34: 829-837. doi: 10.1590/ s0103-84782004000300027

6. Bustamante L, Sulbaran M, Sakai C, de Moura E, Bustamante-Perez L, Nahas C, Nahas $S$, et al. 2016. Una técnica novedosa para corrección de prolapso rectal completo: rectopexia percutánea asistida por endoscopia con ayuda del EndoLifter. Rev Gastroenterol Mex 81: 202-207. doi: 10.1016/j.rgmx.2016.04.004

7. Corgozinho K, Belchior C, De Souza $H$, Ferreira AM, Resende C, Damico B, Cunha S. 2010. Silicone elastomer sling for rectal prolapse in cats. Canadian Vet J 51: 506-510.

8. Cunha M, Pelizarri C, Seraffin G, Cunha J, Sampaio K, Sousa R, Pippi $N$. 2015. Prolapso retal associado a divertículo vésico-uracal em gato. Ciência Animal 25: 35-39.

9. Czajkowski P, Fryer KJ. 2020. Colonic torsion in 4 Great Danes. J Vet Emerg Crit Car 30: 581-586. doi: 10.1111/ vec. 12986

10. Demetriou JL, Welsh EM. 1999. Rectal prolapse of an ileocaecal neoplasm associated with intussusception in a cat. J Feline Med Surg 1: 253-256. doi: 10.1053/jfms. 1999.0041

11. Goodall SV, Chinnadurai SK, Kwan T, Aitken-Palmer C. 2018. Surgical treatment of recurrent rectal prolapse in an adult female black-crested mangabey (Lophocebus aterrimus) by colopexy. Comp Med 68: 80-83.

12. Hernández C. 2010. Emergencias gastrointestinales en perros y gatos. Rev CES Med Vet Zootec 5: 69-85.
13. Jayaprakash $R$, Das B, Sooryadas $S$, Kumar R. 2010. Colopexy with entroplication for recurrent rectal prolapse in a cat. Indian Vet J 87: 279-280.

14. Oliveira G, Oliveira $C$, Raiser A, Silva $S$, Mônaco F. 2009. Colopexia em ovinos da raça Dorper com prolapso retal. Ciênc Rural 39: 479-483. doi: 10.1590/s0103-84782009000200025

15. Popovitch CA, Holt D, Bright R. 1994. Colopexy as a treatment for rectal prolapse in dogs and cats: a retrospective study of 14 cases. Vet Surg 23: 115-118. doi: 10.1111/j.1532-950x.1994.tb00455.x

16. Rockbrand L, Rojas H, Pizarro V, Arias D, Arguedas M. 2019. Manejo quirúrgico del prolapso rectal: una revisión de la literatura. Rev Clín Escuela Med UCR-HSJD 9: 11-20.

17. Sánchez I, Agudelo K, Cuenca W, Joven F, Gonzalez L, Marroquín L, Perdomo L. 2017. Corrección de prolapso rectal en canino Bulldog: a propósito de un caso clínico. REDVET 18(11). [Internet]. Disponible en: https://www.redalyc.org/pdf/636/63653574-025.pdf

18. Secchi P, Filho HC, Scussel Feranti JP, de Oliveira MT, Gottlieb J, Guedes $R L$, et al. 2012. Laparoscopic-assisted incisional colopexy by two portals access in a domestic cat with recurrent rectal prolapse. J Feline Med Surg 14: 169-170. doi: $10.1177 / 1098612 X 11429230$

19. Smeak DD. 2020. Colopexy. In: Gastrointestinal surgical techniques in small animals. John Wiley. p 231-233.

20. Torrijo Gómez I, Balciscueta Coltell $Z$, Sanjuan Jiménez J, Uribe Quintana N. 2018. Solitary rectal ulcer in a patient with rectal prolapse. Cir Esp (Engl Ed) 96: 234. doi: 10.1016/ j.ciresp.2017.08.012.

21. Williams J. 2009. Manual de cirugía abdominal en pequeños animales. JM Sastre Vida. 450 p. 